\title{
Les organisations internationales au cour des crises
}

\author{
Configurations empiriques et jeux d'acteurs
}

\section{David Ambrosetti et Yves Buchet de Neuilly}

\begin{abstract}
Conflits armés, catastrophes naturelles, crises financières, effondrements étatiques... Les « crises » aux formes diverses (et souvent contestées), infranationales, nationales ou internationales, sont aujourd'hui l'objet d'interventions croissantes d'organisations internationales. ONU, HCR, OTAN, FMI, FAO, UE, OSCE, PNUD, BM, G8, AIEA, UA... La liste serait bien longue, et l'on pourrait y ajouter tous les dispositifs ad hoc (Groupes de contact, Quartet, etc.), créés le temps d'une crise, pour la crise dont ils contribuent à dessiner les contours ${ }^{1}$.
\end{abstract}

Il est important de comprendre pourquoi et comment ces organisations diverses investissent de telles « crises », se saisissent ainsi parfois d'enjeux nouveaux et consolident ou étendent, ce faisant, leurs champs d'action respectifs. L'interrogation est résolument spécifique, contextuelle, appelant une enquête empirique au cas par cas. Mais il ne s'agit pas de renoncer à toute ambition théorique pour autant. Une telle bureaucratisation internationale ne reste probablement pas sans effet sur la composition des acteurs en présence dans les domaines concernés, et sur la nature des relations de pouvoir entre ces acteurs, Etats inclus. Jusqu'où ? En quoi les organisations internationales produisentelles un environnement spécifique, distinct d'autres configurations politiques internationales? En quoi contribuent-elles à tracer de nouvelles lignes dans le jeu, et de nouvelles règles du jeu ? De telles transformations (ou leur absence) doivent être établies à partir d'un dialogue entre concepts et matériaux empiriques.

1. Ce dossier prolonge un colloque organisé le 16 octobre 2008 à l'Institut des Sciences sociales du Politique, Université Paris Ouest Nanterre La Défense, dans le cadre du Groupe de recherche du CNRS «Crises extrêmes » (GDR 2651, CRPS, Université Paris I), animé par Johanna Siméant. Les coordinateurs de ce dossier tiennent ici à remercier Johanna Siméant et les membres du GDR pour leur soutien. 
Parfois très élaborées, les théories des relations internationales généralement utilisées pour répondre à ces questions se placent à un niveau de généralisation très élevé. Elles tendent ainsi à enfermer le chercheur dans un maillage serré d'axiomes et d'hypothèses, conséquence nécessaire de la forte ambition nomologique qui les caractérise. Aussi louables soient ces entreprises, on admettra néanmoins qu'elles ont pour effet d'éloigner le chercheur de l'observation attentive et de longue durée de l'objet étudié - ici les organisations internationales -, de perdre de vue les apports attendus d'une démarche inductive fructueuse.

Les approches dominantes, en particulier néoréalistes, font peu de cas des organisations internationales, réduites au rang de simples instruments des Etats ${ }^{2}$. Même les institutionnalistes néolibéraux, attachés à un axiome intergouvernementaliste irréductible, limitent bien souvent l'apport et l'impact de ces organisations à quelques économies potentielles de coûts de transactions ${ }^{3}$. Les organisations internationales ne seraient qu'un instrument à disposition des Etats. Elles seraient tributaires de leurs rapports de force qui non seulement en produiraient les contours, mais décideraient de surcrôit de leurs (non) usages. Deux difficultés majeures apparaissent aussitôt : tout se passe comme si les organisations internationales ne pouvaient faire autre chose que ce pour quoi elles auraient été créées (ou renégociées ensuite par les Etats); tout se passe comme si les Etats étaient des entités cohérentes avec des préférences stables au niveau international, et donc dans les organisations internationales.

Le problème de l'autonomie et des intérêts propres des organisations internationales cristallise en effet les oppositions entre spécialistes et écoles des relations internationales, que ce soit sur les régimes internationaux ${ }^{4}$ ou sur les processus d'intégration régionale, et particulièrement la construction des communautés puis de l'Union européenne ${ }^{5}$. Certes, contrairement aux réalistes, les libéraux n'appréhendent pas l'Etat comme une boîte noire, et l'intérêt national comme un donné structurel (lié à la position de l'Etat dans le système politique international). Mais si les concurrences d'intérêts sont bien à

2. Voir par exemple Grieco J., «Anarchy and the Limits of Cooperation: A Realist Critique of the Newest Liberal Institutionalism ", International Organization, vol.42, n³, été 1988, p. 485-508.

3. Keohane R. O., After Hegemony, Princeton, Princeton University Press, 1984, ou Martin L. L. and Simmons B. A., "Theories and Empirical Studies of International Institutions », International Organization, vol.52, ${ }^{\circ} 4$, automne 1998, p. 729-757.

4. Hasenclever A., Mayer P., Rittberger V., Theories of International Regimes, Cambridge et New York, Cambridge University Press, 2002 (5e éd.).

5 . D’abord inspirés par la théorie économique de l'agence (la relation entre les mandants [principal], ici les Etats, et les mandataires [agent], ici les organisations internationales) qui recherchait les voies d'optimisation du contrôle des mandataires (agent) par leurs mandants (principal), les spécialistes des relations internationales ont dû rivaliser avec les apports des théories des politiques publiques pour étudier les communautés puis l'Union européennes. Pour une synthèse théorique, voir notamment Rosamond B., Theories of European Integration, Basingstoke et New York, Macmillan et St. Martin's Press, 2000. Les libéraux, en particulier intergouvernementalistes sous la plume d'Andrew Moravcsik, n'ont pas pour autant cessé 
l'œuvre au sein des Etats, le gouvernement (au sens large) est pensé comme un mécanisme d'arbitrage et de centralisation des revendications ${ }^{6}$. Il constitue dès lors une entité stable et cohérente dans les arènes multilatérales.

Pour échapper à cette naturalisation de l'acteur étatique et de ses intérêts, certains auteurs se sont replongés dans les approches plus critiques de l'ordre international questionnant les logiques hégémoniques des Etats, en particulier dans le domaine des idées, des valeurs et des normes qui dominent au sein des organisations internationales 7 . Depuis quelques années, dans la littérature scientifique en relations internationales, les approches regroupées sous le label «constructiviste» fournissent les instruments d'analyse les plus fréquemment utilisés pour soutenir l'hypothèse de l'autonomie de ces organisations, ou à tout le moins celle de la spécificité du jeu politique international qui s'y déroule. Elle mesure, pour ce faire, la reconfiguration des idées (idée de soi, des autres, de la situation rencontrée, de la chose à faire) dans le cours même de l'interaction multilatérale, et ses conséquences sur les formes d'exercice de la puissance par les Etats, sur leurs dispositions à coopérer ou non, et sur les stratégies hégémoniques qui en découlent ${ }^{8}$.

Dans ces travaux, toutefois, le souci de contrecarrer le néo-réalisme et de démontrer les espaces possibles de coopération et leurs vertus tend à supplanter l'attention portée aux organisations internationales pour ce qu'elles sont, dans leurs dimensions les plus concrètes, quotidiennes. Par exemple, la prise en compte des «communautés épistémiques » qui portent les idées dominantes au sein de ces organisations renvoie trop rapidement aux nouvelles chances de coopération censées découler de la montée en force de ces experts ${ }^{9}$, plutôt qu'aux logiques concrètes de repositionnement de la part des décideurs politiques et diplomatiques face à ces communautés, en fonction des groupes sociaux plus précis auxquels ils appartiennent, qui tantôt investissent, tantôt combattent ces savoirs experts dominants. Et l'on pourra adresser le même reproche à la perspective développée par Michael Barnett et Martha

d'affirmer la prééminence des Etats (eux-mêmes reflétant les préférences de leurs sociétés nationales) et l'impossibilité pour les organisations internationales (ici l'Union européenne et ses administrations supranationales, notamment la Commission) de s'affranchir de leur tutelle étatique. Voir en particulier Moravcsik A., The Choice for Europe: Social Purpose and State Power from Messina to Maastricht, Ithaca, Cornell University Press, 1998.

6. Evans P. B., Jacobson H. K., et Putnam R. B. (dir.), Double-Edge Diplomacy: International Bargaining and Domestic Politics, Berkeley, University of California Press, 1993.

7. Cox R., avec Sinclair T., Approaches to World Order, Cambridge, Cambridge University Press, 1996.

8. Ruggie J. G. (dir.), Multilateralism Matters: The Theory and Praxis of an Institutional Form, New York, Columbia University Press, 1993; Cronin B., «The Paradox of Hegemony: America's Ambiguous Relationship with the United Nations », European Journal of International Relations, vol.7, $\mathrm{n}^{\circ} 1$, 2001, p. 103-130 ; Johnston A. I., « Treating International Institutions as Social Environments ", International Studies Quarterly, vol.45, n 4,2001 , p. 487-515.

9. Haas P., «Epistemic Communities and International Policy Coordination », International Organization, vol.46, ${ }^{\circ} 1,1992$, p. 1-35. 
Finnemore, consistant à appliquer à l'international la sociologie wébérienne de la bureaucratie ${ }^{10}$ : trop rapidement, le regard quasi ethnographique et hautement réflexif proposé par Barnett dans son célèbre article sur le rôle d'expert qu'il a dû apprendre à jouer à la Mission permanente des Etats-Unis à l'ONU lors du conflit et du génocide rwandais (1990-1994) ${ }^{11}$ disparaît dans l'effort de systématisation de l'analyse et de fabrication d'une boîte à outils alternative au néo-réalisme, logiques de positionnement internes au champ universitaire étatsunien obligent.

Reste enfin tout un pan de la littérature consacré aux réponses aux crises d'un point de vue essentiellement opérationnel (ou problem-solving), qui ne retient les éléments propres aux organisations internationales que dans la mesure où ces éléments peuvent être érigés en facteurs généralisables de succès ou d'échec dans la résolution de ces crises, dans l'efficacité de l'action de ces organisations ${ }^{12}$.

C'est armés de questionnements davantage sociologiques et de riches données empiriques que les auteurs de ce dossier nous donnent à voir ce que font et ce que fondent les organisations internationales, à différents niveaux, et entrent en résonance avec certains débats très actuels de la théorie internationaliste, essentiellement inspirés par les approches critiques de la discipline.

Deux ensembles de questions sont ainsi soulevés.

1/ En premier lieu, pourquoi et comment une situation politique est-elle perçue et construite comme une crise potentiellement « gérable » en tout ou partie par des institutions multilatérales ? Qu'apporte la qualification « crise » aux organisations internationales, en termes de solidification institutionnelle, de capacité d'expansion de leurs activités, mais aussi en termes de contraintes et de risques nouveaux?

Si l'on s'intéresse ici à des « crises » aussi différentes que les catastrophes naturelles, les conflits armés, le chômage, c’est bien évidemment pour échapper à toute tentation substantialiste, pour souligner que la «crise » est avant tout une catégorie pratique, un enjeu et une contrainte (lorsque la qualification sociale se durcit ou qu'il s'agit de la durcir). Le terme «crise » n'est guère plus précis que celui de "problème » de politique publique, auquel les acteurs voudraient donner une priorité politique, tout en renvoyant à une forme de

10. Barnett M. N. et Finnemore M., «The Politics, Power, and Pathologies of International Organizations ", International Organization, vol.53, n4, 1999, p. 699-732; Rules for the World: International Organizations in Global Politics, Ithaca, Cornell University Press, 2004.

11. Barnett M. N., «UN Security Council, Indifference, and Genocide in Rwanda », Cultural Anthropology, vol.12, n 4, 1997, p. 551-578.

12. Exemple parmi tant d'autres, Howard L. M., UN Peacekeeping in Civil Wars, Cambridge, Cambridge University Press, 2008. 
légitimité de l'intervention publique. Dans bien des cas, qualifier une situation de crise appelle et autorise l'action publique (quand bien même cette qualification serait ou non l'enjeu d'un débat ${ }^{13}$ ).

Une organisation comme l'OTAN n'était pas nécessairement « destinée » à prendre en charge des «crises internationales ", i.e. à intervenir sous ce label en dehors du périmètre géographique et opérationnel qui lui avait été assigné pendant la guerre froide. Christophe Wasinski montre comment un vaste travail cognitif a rendu possibles et pensables à la fois la « gestion de crises » et le recours à une organisation internationale comme l'OTAN pour le faire. On découvre que ce travail ne passe pas seulement par les discours politiques et diplomatiques déployant une série d'arguments de principes et d'opportunité, mais tout autant par la diffusion d'un «sens commun technique », d'une conviction technique parmi les personnels de l'organisation ainsi que les groupes d'acteurs associés aux fins de l'OTAN, conviction selon laquelle « la gestion de crise, ça marche ! ». Et si la « crise » semble s'offrir aux organisations internationales telles que l'OTAN comme une exception requérant en soi une autre échelle que celle strictement nationale/étatique, la quête technique qui en découle plonge, elle, dans la pensée stratégique proprement étatique, étatsunienne en l'occurrence, selon des logiques d'attraction et de diffusion propres aux professionnels de la sécurité et de la stratégie militaire qui restent à interroger.

Cette capacité à agir, qui s'exprime en mots et en actes, excède largement le cas des conflits interétatiques de haute intensité. Bien des «crises » - et le flou de la catégorie a ici toutes ses vertus - peuvent être gérées par l'OTAN, y compris des situations de « crise » qui ne nécessitent pas, ou très marginalement, le recours à des moyens coercitifs. Sandrine Revet suit ainsi le cheminement de l'intervention de l'OTAN en 2005 aux Etats-Unis après le passage de l'ouragan Katrina et au Pakistan après le tremblement de terre, qui sont autant d'occasion de tester, de valider et d'imposer les nouveaux dispositifs produits par le Centre Euro-atlantique de coordination des actions en cas de catastrophe (EADRCC). Ces nouveaux investissements de l'OTAN participent d'un processus plus vaste de recomposition et de transformation du champ d'intervention «sécuritaire » international dans l'après guerre froide. Les frontières entre actions civiles et militaires sont retravaillées. Au nom de la cohérence, ces dispositifs doivent s'intégrer, faisant naître ou intensifiant des tensions entre les professionnels de la sécurité et les professionnels de l'action humanitaire et du développement, entre des instruments standardisés et des dispositifs individualisés. Sandrine Revet offre ainsi une véritable cartographie de ce champ d'intervention, de cette gestion globale des risques et des désastres naturels en cours de constitution.

13. Buchet de Neuilly Y., « La crise ? Quelle crise ? Dynamiques européennes de gestion des crises ", in Le Pape M., Siméant J., Vidal C. (dir.), Crises extrêmes. Face aux massacres, aux guerres civiles et aux génocides, Paris, La Découverte, 2006, p. 270-286. 
Dans un autre domaine, celui de la lutte contre le chômage, Vincent Gayon retrace les enjeux des positionnements successifs d'une organisation telle que l'OCDE en situation d'expertise économique face à une « crise » qui frappe ses Etats membres au tournant des années 1990. La quête de légitimité entreprise par cette organisation internationale gagne ainsi à être resituée à l'aune des logiques de fonctionnement et d'une culture propres à ses producteurs d'expertise économique, mais aussi des formes de coproduction et de réception de cette expertise par la principale «clientèle » de l'OCDE, les gouvernements nationaux et les diplomates qui les représentent, sans oublier ici les efforts destinés aux audiences médiatiques de ces gouvernements de l'OCDE. De l'expertise face à la crise de l'expertise, les tactiques argumentatives et les ressources relationnelles mobilisées par l'organisation se dévoilent au fil de ses contorsions, pour que perdure la crédibilité de son offre d'expertise. On voit bien que la production du possible, du pensable et du crédible ne dépend pas seulement des multiples investissements dans l'organisation internationale ou des organisations internationales. Celles-ci sont dépendantes de transformations sectorielles plus larges, d'une évolution plus ou moins forte des structures de plausibilités, ou pour reprendre les termes de Vincent Gayon, de déplacement du point de crédibilité des remèdes à la crise. L'action des organisations internationales s'insère dans des imbrications d'arènes, nationales et supranationales, où les organisations internationales ne sont pas toujours présentes, ou lorsqu'elles le sont, avec un poids très variable.

2/ Cette prise en compte des «transactions collusives » 14 - par lesquelles une organisation internationale comme l'OCDE et les représentants des gouvernements qui la composent ajustent au quotidien les expertises produites aux diverses logiques institutionnelles en présence - conduit à un second ensemble de questions : pourquoi et comment les membres des organisations internationales, les bureaucraties supranationales, les tiers (destinataires directs ou non de l'éventuelle opération) interpellent-ils les organisations internationales ? Quelles sont les conditions du succès ou de l'échec de la mise sur agenda ? Autrement dit, qu'induit « un passage par l'organisation internationale » pour les acteurs, étatiques et autres, qui décident de porter une question au sein d'une organisation internationale, ou qui sont amenés à prendre position, à participer à la gestion d'une telle question qualifiée de « crise », en somme pour les différents acteurs qui font agir les organisations internationales et/ou agissent en leur sein ? Quelles spécificités peut-on en déduire à propos de la politique internationale qui prend place dans cet environnement spécifique que constitue une organisation internationale?

Contrairement à l'image spontanée que ces organisations donnent d'ellesmêmes, reposant sur une définition procédurale de l'action, ces processus ne sont pas séquentiels. La « crise » n’est pas nécessairement un préalable à l'im-

14. Dobry M., Sociologie des crises politiques, Paris, Presses de Sciences Po, 2009 (3ème édition). 
pulsion d'une action. Les préférences et même les intérêts à agir ne sont pas tous directement connectés aux évènements pris en charge. Et de surcroît, ils peuvent se redéfinir dans le cours de l'action. Il s'agirait dès lors d'identifier les dynamiques de négociation, les logiques d'investissement dans le jeu, la (re)construction des stratégies - voire même des positions -, les modalités d'alliances et les ressources mobilisables dans ces arènes bien souvent imbriquées les unes aux autres.

A travers le cas de la diplomatie française à Bruxelles, Yves Buchet de Neuilly retrace les implications, pour les représentants des Etats concernés, d'un investissement au sein d'une organisation internationale en termes de socialisation professionnelle, de stratégies de carrière, de compétences requises ou prêtées. Ce sont là des logiques sociales, rarement soulignées, de la construction des intérêts que les Etats portent face aux problèmes discutés au sein des organisations internationales. En quittant leur capitale pour atteindre la ligne de front des négociations multilatérales, les diplomates chargés de représenter l'Etat dans les arènes décisionnelles centrales des organisations internationales sont soumis à de puissantes contraintes de coordination. Confrontés aux attentes, parfois contradictoires et antagonistes, pesant sur la nouvelle position qu'ils occupent dans le secteur d'action publique où se déploie l'activité de l'organisation internationale, les diplomates doivent rapidement apprendre à gérer leur insertion dans des jeux multiples, interdépendants, se déroulant simultanément dans et entre les capitales, dans et entre les sièges des organisations internationales. La maîtrise des dossiers, comme dans tout autre secteur de l'action publique internationale, se traduit d'abord par un apprentissage accéléré de l'univers du possible, des coups autorisés, de l'inertie des routines organisationnelles. En s'appropriant ces nouveaux savoir-faire, en gérant avant tout la complexité de leur position, les diplomates socialisés aux «bonnes pratiques » européennes de gestion de crises n'expriment en rien une quelconque adhésion au «projet » de l'organisation internationale ou un quelconque renoncement à la confrontation des «intérêts ».

A propos de ces «intérêts » précis portés par les représentants des Etats en matière de régulation des conflits armés à l'ONU, David Ambrosetti expose la portée des règles formelles et surtout pratiques (ou pragmatiques) qui sont actualisées et reproduites par les délégations diplomatiques au sein du Conseil de sécurité. On voit ici combien les positions des représentants diplomatiques - dans les deux sens du terme "position » : leurs préférences affichées et les positions sociales et professionnelles qu'ils occupent au sein de leur hiérarchie et vis-à-vis de leurs pairs - continuent de dépendre d'enjeux élaborés en dehors du temps de l'urgence provoquée par les «crises » discutées, et en partie à l'extérieur du Conseil de sécurité. Mais ces positions s'ajustent à la grammaire du jeu multilatéral ainsi investi par les Etats face à des audiences plus larges. En découle un ensemble de règles, de routines et de 
positions informelles nouvelles reconnues par les diplomates, dès l'inscription à l'ordre du jour du Conseil puis dans les négociations en vue de l'adoption d'un texte, c'est-à-dire d'une décision du Conseil. Ces règles sont sources de nouvelles opportunités mais aussi de nouveaux risques dans l'exercice de leur fonction, et même dans le déroulement de leur carrière. La prise en compte de ces risques précis attachés à des positions sociales informelles précises - telle que celle de leader d'un dossier - conduit ainsi à mieux comprendre les dispositions des délégations membres du Conseil à se saisir d'une situation de conflit armé et à l'ériger en crise.

Vincent Gayon montre également comment certaines formes propres aux organisations internationales d'expertise façonnent les types d'investissement et les outils requis de la part des délégations des Etats. Ces dernières se trouvent engagées dans la coproduction d'études, dans la définition de critères de comparaison, de classements, ce qui ne les conduit pas à bannir définitivement les arguments strictement politiques et idéologiques face aux choix que l'économie libérale a permis de naturaliser et techniciser au sein de groupes professionnels experts. Cela renvoie à la question des logiques de circulation des individus et des concepts et techniques entre organisations (étatiques, internationales, non gouvernementales), que pose Sandrine Revet à propos des acteurs qui ont su gagner une visibilité suffisante dans le domaine de la réponse aux catastrophes naturelles et de la réduction des « risques naturels ».

Le présent dossier entend ainsi nourrir une sociologie des acteurs engagés dans l'expertise et la négociation au sein des organisations internationales, une connaissance accrue de nouvelles formes de professionnalisation, de nouvelles carrières de "gestionnaires » de crises, de leurs routines, notamment en matière de division du travail entre organisations et entre catégories de professionnels (militaires, magistrats, diplomates, policiers, gendarmes et autres « experts»), aussi de leurs stratégies de mobilisation, et enfin des effets de ces différents processus sur la construction des problèmes et des solutions internationales 15 .

15. On pensera ici également au récent ouvrage publié par Amandine Scherrer, G8 Against Transnational Organized Crime, Burlington, Surrey, Ashgate, 2009, qui confirme toute la portée de cette approche sociologique appliquée aux organisations internationales et aux professionnels de la diplomatie et autres experts qui les composent. 